\title{
ÉDITORIAL
}

\section{Révolution ou évolution?}

Le temps passe si vite et si lentement à la fois. Autant la place de l'humain au travail a pris des décennies à se définir, autant elle s'est construite sous de nouvelles formes en modifiant la nature du travail et ses composantes. L'emprise du temps fait son œuvre. Le travail n'a jamais été si complexe en raison de la demande sans cesse grandissante du niveau de compétences requises pour accomplir les obligations professionnelles. La courbe d'imputabilité des travailleurs a progressé au même rythme, bousculant au passage une main-d'œuvre souvent affectée par le stress et la pression afférente. Une relecture de la croisade de l'humain au travail met en relief les entraves à l'obtention d'un point d'équilibre entre la complexité des tâches à effectuer et la performance attendue. Bref, le temps n’a rien arrangé, il a plutôt mis en exergue les reliefs discrets, et souvent épineux, de la relation des travailleurs avec les tissus organisationnels.

L'objet de cet appel à contribution, la quatrième révolution industrielle et l'humain au travail, s'inscrit dans cette quête incessante d'une meilleure compréhension des dynamiques organisationnelles. Cette quête passe forcément par l'étude du changement sous toutes ses formes et de son impact sur les acteurs en cause. En effet, ces derniers appréhendent leur univers en fonction de leur référent, mais également en fonction des sources d'influences externes qui modulent les nouveaux environnements de travail. Ainsi, l'identification des changements individuels et collectifs devient un champ d'intérêt passionnant non seulement pour les chercheurs, mais également pour les praticiens réflexifs en quête d'un nouveau monde du travail. C'est donc avec curiosité et enthousiasme que ce deuxième numéro de la revue Ad machina vous est présenté.

Toute la complexité de l'évolution de l'humain au travail ne pourrait se résumer dans les articles qui composent ce numéro. Toutefois, la façon dont les auteurs ont mis en évidence leurs objets de recherche, à la fois contemporains et lucides, jette un éclairage pertinent et appelle à la réflexion. Des thèmes tels que la gestion de projet dans les industries créatives, les travailleurs et la révolution 4.0, la main-d'œuvre proche aidante sous le prisme des temps sociaux, l'ubérisation des marchés financiers, l'ère du numérique et de l'économie collaborative ainsi que l'instrumentalisation de la pleine conscience constituent une illustration éloquente des efforts déployés par la revue afin d'augmenter notre compréhension des enjeux sociaux, économiques et humains avec la rigueur requise et attendue par l'ensemble de la communauté scientifique. Il convient, à cet effet, de remercier les contributeurs de ce numéro et d'inviter les chercheurs et créateurs à collaborer aux numéros à venir dans le but de permettre à la recherche sur l'humain au travail de se démarquer par son audace, sa créativité et sa curiosité. La revue Ad machina se veut un vecteur d'idées, dans un format numérique en libre accès, et ce, pour favoriser le partage et la profondeur du travail déployé par ses collaborateurs. Il est d'usage de terminer en vous souhaitant une bonne lecture.

Le comité éditorial,

\section{Olivier Gagnon, Université du Québec à Chicoutimi \\ Stéphanie Maillet, Université de Moncton \\ Eric Jean, Université du Québec à Chicoutimi}

Doi: 10.1522/radm.no1.922 